\title{
Hypertonic saline for prevention of delirium in geriatric patients who underwent hip surgery
}

\author{
$\mathrm{Xi} \mathrm{Xin}^{1 \dagger}$, Fei Xin ${ }^{2 \dagger}$, Xuguang Chen ${ }^{1}$, Qi Zhang ${ }^{1}$, Yanan $\mathrm{Li}^{1}$, Shuping Huo ${ }^{1}$, Chongfu Chang ${ }^{1}$ and Qiujun Wang ${ }^{1 *}$ (D)
}

\begin{abstract}
Background: Postoperative delirium (POD) is a common disorder in the elderly patients, and neuroinflammation is the possible underlying mechanism. This study is designed to determine whether or not hypertonic saline (HS) pre-injection can alleviate POD in aged patients.

Methods: This prospective study recruited 120 geriatric patients who underwent hip surgery. The patients were randomly divided into two groups: control group (NS group) and HS group. Patients in the NS group were pre-injected with $4 \mathrm{~mL} / \mathrm{kg}$ isotonic saline, and those in the HS group were pre-injected with $4 \mathrm{~mL} / \mathrm{kg} 7.5 \% \mathrm{HS}$. All 120 patients were then subjected to general anesthesia. Blood samples were extracted to detect the concentration of inflammatory factors, namely, IL-1 $\beta, I L-6, I L-10$, and TNF-a, and the nerve injury factor S100ß. Flow cytometry was used to detect the number of monocytes in peripheral venous blood and evaluate the relationship of inflammation to delirium. The nursing delirium screening scale (Nu-DESC) was used to determine cognitive function 1 to 3 days postoperatively.

Results: Analysis using random-effect multivariable logistic regression indicated that $\mathrm{HS}$ administration before anesthesia was associated with a low risk of POD (odds ratio [OR], $0.13 ; 95 \% \mathrm{Cl}, 0.04$ to $0.41 ; P=0.001$ ) and few $C D 14+$ CD16+ monocytes ( $\beta=-0.61 ; 95 \% \mathrm{Cl},-0.74$ to $-0.48 ; P=0.000$ ) the following day. When the association between HS and delirium was controlled for CD14 + CD16+ monocytes, the effect size became nonsignificant (odds ratio [OR], 0.86; $95 \% \mathrm{Cl}, 0.14$ to 5.33; $P=0.874$ ). TNF-a was significantly associated with POD (odds ratio [OR], 1.10; 95\% Cl, 1.05 to 1.16; $P=0.000)$. However, IL-1 $\beta, I L-6, I L-10$, and $S 100 \beta$ were not significantly related to POD.
\end{abstract}

Conclusion: $\mathrm{HS}$ can alleviate POD in geriatric patients and may inhibit the secretion of inflammatory factors by monocytes.

Keywords: Hypertonic saline, Postoperative delirium, Elderly, Monocytes, Cytokines, Neuroinflammation

\section{Background}

Postoperative delirium (POD) is a common acute cerebral disorder that is characterized by sudden and transient disturbances in awareness, consciousness, and cognition $[1,2]$. POD is an age-related syndrome, primarily occurring in the elderly population ( $>65$ years of age) and may be associated with increased morbidity, length of hospital stay, and patient care costs [3]. The highest incidence of POD is found in patients with hip fractures (35-65\%) particularly 3 days after the operation [4], since the

\footnotetext{
* Correspondence: 18713488965@163.com

${ }^{\dagger}$ Equal contributors

'Department of Anesthesiology, The Third Hospital of Hebei Medical University, No 139, Ziqiang Road, Shijiazhuang 050051, Hebei, People's Republic of China

Full list of author information is available at the end of the article
}

fracture itself and subsequent surgery are two events leading to a systemic response. Elderly patients with preoperative cognitive impairment, dementia, anxiety, depression, and malnutrition are at great risk of POD [5]. The pathophysiology of POD has not been completely understood, and no specific treatments are currently available.

Neuroinflammation plays an important role in the development of delirium [6, 7]. Aseptic surgery increases in inflammatory markers in serum and causes inflammationmediated, hippocampal-dependent, and cognitive dysfunctions. Increased serum levels of inflammatory cytokines postoperatively are associated with memory impairment, reactive microgliosis, and upregulated interleukin (IL)-1 $\beta$ expression in the hippocampus [6]. Inflammatory cytokines are mainly involved in postoperative cognitive delirium [8]. 
Cytokines are categorized as inflammatory cytokines, including IL-1 $\beta$, IL-6, IL-8, and tumor necrosis factor alpha (TNF- $\alpha$ ), and anti-inflammatory cytokines, such as IL-10. Inflammatory cytokines may lead to the occurrence and development of diseases, that is, high levels of inflammatory cytokines are related to the severity of the disease $[9,10]$. The role of systemic TNF- $\alpha$ has been widely investigated; a high level of systemic TNF- $\alpha$ is associated with twofold increase in disease symptoms, including apathy, anxiety, depression, and agitation [11]. CD14+CD16+ monocytes exhibit enhanced capacity for TNF- $\alpha$ generation [12]. Thus, restraining and regulating inflammatory factors may improve the prognosis of delirium.

Hypertonic saline (HS) is widely used as resuscitation fluid in patients with traumatic hemorrhagic shock. HS exerts not only beneficial effects on hemodynamic parameters but also modulatory effects on various immune cell functions, such as degranulation, adhesion, cytokine expression, and reactive oxygen species production [13]. HS modulates local and systemic inflammatory response $[14,15]$, but its effect on alleviating postoperative delirium remains to be discussed.

This study aims to investigate the effect of HS on elderly patients with POD and preliminarily determine the relationship among HS, neuroinflammation, and POD. Results may provide reference for clinical applications. We tested the hypothesis that prophylactic HS could alleviate delirium and could be related to reduction of systemic inflammation.

\section{Methods}

\section{Patients and setting}

This prospective cohort study recruited patients ( $>65$ years old) who underwent hip arthroplasty for femoral neck fracture surgery in the Third Hospital of Hebei Medical University in July 2017. We collected basic patient information, including age, sex, body mass index (BMI), American Society of Anesthesiologists (ASA) score, coronary artery disease, hypertension, diabetes, and cerebral infarction. The mini-mental state examination (MMSE) was administered to screen for preoperative cognitive function 1 day before the surgery. The exclusion criteria were as follows: ASA physical status of above III, age of 65 and younger, MMSE score of less than 24 or dementia due to various etiologies, preoperative delirium, history of neurological or mental illness, current use of tranquilizers or antidepressants, history of an endocrine or metabolic disorder, recent use of glucocorticoids or other hormones, suffering from infections or chronic inflammatory conditions, intake of anti-inflammatory drugs, unwillingness to complete the experimental procedures, presence of a language barrier, severe hearing or visual impairment, illiteracy, and alcohol or drug dependence. Only 120 patients were enrolled in the study. The patients were divided into two groups $(n=60)$ by using a random number table: 7.5\% HS group and normal saline group (NS group). All patients were required to provide written informed consent before randomization. If acute heart failure, renal failure, and electrolyte disorder occurred during the treatment, then the experiment was terminated and relevant measures were performed.

\section{Anesthesia and surgical management}

Invasive blood pressure, pulse oxygen saturation, electrocardiogram, bispectral index, and end-tidal $\mathrm{CO}_{2}\left(\mathrm{ETCO}_{2}\right)$ were continuously monitored in the two groups during the perioperative period. In brief, $4 \mathrm{~mL} / \mathrm{kg} 7.5 \% \mathrm{HS}$ (Shijiazhuang No. 4 Pharmaceutical Co., Ltd.) or NS was injected $30 \mathrm{~min}$ before administering anesthesia.

Intraoperative circulatory system was maintained by adjusting the anesthesia depth (controlling BIS 40 to 60) and administering transfusions or cardiovascular agents as necessary. Blood pressure fluctuation amplitude did not exceed $20 \%$ of the base value. Scopolamine and penehyclidine were prohibited. Atropine was used only to reverse bradycardia. All patients' surgery time were controlled within hours. After the surgery, all of the patients were transferred to surgical intensive care unit and received the same treatment.

\section{Blood sample collection and sample bank establishment} Venous blood was drawn at 06:00 on the first day after the surgery. Blood samples were immediately placed into sterile EDTA test tubes and centrifuged at $3000 \mathrm{~g}$ for $30 \mathrm{~min}$ at $4{ }^{\circ} \mathrm{C}$ to collect plasma. Plasma was aliquoted into polypropylene tubes and stored in a freezer at $-80^{\circ}$ $\mathrm{C}$ (Sanyo, Japan) until further assayed. Additional venous blood was collected for flow cytometry to determine the number of monocytes.

\section{Cytokine measurement}

The concentrations of IL-1 $\beta$, IL-6, TNF- $\alpha$ (pro-inflammatory cytokines), IL-10 (anti-inflammatory cytokine), and $\mathrm{S} 100 \beta$ in plasma were quantified with a commercial enzyme-linked immunosorbent assay (ELISA) kit (Duoset, R\&D Systems, UK) in accordance with the manufacturer's instructions. ELISA assays were read by SoftMax Pro version 5.3. All samples were assayed in duplicate. The person carrying out the assays was completely blinded to the clinical information.

\section{Blood monocyte subset redistribution}

The samples were further stained with anti-CD16-PE and anti-CD14-FITC mAbs (BD Biosciences, San Jose, CA, USA) to identify monocyte subsets. Class-matched isotype immunoglobulin negative control mAbs (BD Biosciences, San Jose, CA, USA) was added to separate tubes for the same samples to detect nonspecific 
binding. The samples were incubated away from light at room temperature for 15 to $30 \mathrm{~min}$. The samples were then incubated with hemolysin until all red blood cells cracked. The plates were washed with $500 \mu \mathrm{L}$ of PBS and centrifuged at $1400 \mathrm{r} / \mathrm{min}$ for $5 \mathrm{~min}$. After the supernatant was removed, the cells were washed again, resuspended in paraformaldehyde, and analyzed by flow cytometry. FACS Calibur software (Beckman-Coulter, USA) was used for cytometric analysis. The analyses were done in duplicate and the technician was blinded to clinical data.

\section{Delirium evaluation}

POD was assessed through $\mathrm{Nu}$-DESC scoring at the same time on the first to third day postoperatively. Five parameters were captured; each with a subscore of $0-2$ points (Table 1). A total of $\geq 2$ points indicated the presence of delirium [16].

\section{Statistical analysis}

All data were analyzed by SPSS (version 21.0 for Windows, SPSS Inc., Chicago, IL, USA). Categorical variables were analyzed through $\chi^{2}$ test or Fisher's exact test and presented as numbers and percentages. Continuous variables were tested with Student's $t$ test for normal distribution or Mann-Whitney $U$ test for skewed distribution and reported as mean \pm standard deviation.

Mediation can be demonstrated using four steps: (1) the effect of the independent variable (HS) on the dependent variable (delirium) must be significant, (2) the path from the independent variable (HS) to the mediator (CD14+ $\mathrm{CD} 16+$ monocytes) must be significant, (3) the path from

Table 1 Nursing delirium screening scale

\begin{tabular}{|c|c|c|c|}
\hline \multirow{2}{*}{$\begin{array}{l}\text { Symptom } \\
1 \text { Disorientation }\end{array}$} & \multicolumn{3}{|c|}{$\begin{array}{l}\text { Symptom } \\
\text { rating }\end{array}$} \\
\hline & 0 & 1 & 2 \\
\hline \multicolumn{4}{|l|}{$\begin{array}{l}\text { Verbal or behavioral manifestation of not being oriented to } \\
\text { time or place or misperceiving persons in the environment }\end{array}$} \\
\hline 2 Inappropriate behavior & 0 & 1 & 2 \\
\hline \multicolumn{4}{|l|}{$\begin{array}{l}\text { Behavior inappropriate to place and/or for the person; e.g., } \\
\text { pulling at tubes or dressings, attempting to get out of bed } \\
\text { when contraindicated, and the like }\end{array}$} \\
\hline 3 Inappropriate communication & 0 & 1 & 2 \\
\hline \multicolumn{4}{|l|}{$\begin{array}{l}\text { Communication inappropriate to place and/or for the } \\
\text { person; e.g., in-coherence, non-communicativeness, } \\
\text { nonsensical or unintelligible speech }\end{array}$} \\
\hline 4 Illusions/Hallucinations & 0 & 1 & 2 \\
\hline \multicolumn{4}{|l|}{$\begin{array}{l}\text { Seeing or hearing things that do not exist; distortions of } \\
\text { visual objects }\end{array}$} \\
\hline 5 Psychomotor retardation & 0 & 1 & 2 \\
\hline $\begin{array}{l}\text { Delayed responsiveness, few or no spontaneous actions/ } \\
\text { words; e.g., when the patient is prodded, the reaction is } \\
\text { deferred and/or the patient is unarousable }\end{array}$ & & & \\
\hline
\end{tabular}

the mediator (CD14+CD16+ monocytes) to the dependent variable (delirium) must be significant, and (4) the independent variable (HS) has a reduced or no effect on the dependent variable (delirium) after adjustment for the mediator (CD14 + CD16+ monocytes) [17].

With steps 3 and 4 tested simultaneously, three mixed-effect logistic regression models were used to test (1) the association between prophylactic HS and delirium the following day, (2) the association between CD14 + CD16+ monocytes and delirium, and (3) the association between prophylactic HS and delirium controlling for CD14+CD16+ monocytes [18]. Another multivariate logistic regression model was employed to determine the association between cytokines and delirium. Associations were assessed with 95\% CI and considered significant at $P$ values less than 0.05 .

\section{Results}

A total of 120 patients who underwent surgery for a femoral neck fracture were enrolled in the study and assigned into two groups. Table 2 shows the main demographic, chronic medical conditions, and the incidence of delirium. No statistically significant differences occurred in the age, gender, BMI, and comorbidities (all $P>0.05$ ). Cytokines and pro-inflammatory monocytes were significant between the two groups $(P<0.01)$. The incidence of delirium was statistically significantly lower in HS group than in NS group $(P=0.001)$.

\section{Association between prophylactic hypertonic saline and delirium}

The independent variable was set as HS (yes or no) administered before anesthesia, and the dependent variable was set as the presence of delirium the following day in the multivariate models to evaluate the effect of HS on elderly patients with POD. In the multivariate analysis model, age, sex, ASA class, BMI, duration of anesthesia, and comorbidities were compared between the non-delirium and total delirium groups. Analysis using logistic regression models indicated HS administration before anesthesia was associated with a low risk of POD (odds ratio [OR], 0.13; 95\% CI, 0.04 to $0.41 ; P=0.001$ ) (Table 3). Furthermore, age, sex, and cerebral infarction remained significant (all $P<0.05$ ). However, the duration of anesthesia, ASA class, MMSE, and other comorbidities were statistically meaningless in relation to delirium.

\section{Association between prophylactic HS and CD14 + CD16+ monocytes}

HS was associated with low levels of CD14+CD16+ monocytes (Fig. 1). The association between HS and CD14 + CD16+ monocytes was assessed using a linear mixed-effect model. The independent variable was set 
Table 2 Baseline demographic variables, subject characteristics, and prevalence of postoperative delirium

\begin{tabular}{|c|c|c|c|}
\hline Variable & Hypertonic saline $(n=60)$ & Normal saline $(n=60)$ & $P$ value \\
\hline \multicolumn{4}{|c|}{ Demographic and clinical characteristics } \\
\hline Age (years) & $76.6 \pm 5.8$ & $75.6 \pm 5.6$ & 0.382 \\
\hline Sex, female (\%) & $31(52)$ & $27(45)$ & 0.534 \\
\hline $\mathrm{BMI}\left(\mathrm{kg} / \mathrm{m}^{2}\right)$ & $28.7 \pm 3.4$ & $28.5 \pm 3.3$ & 0.845 \\
\hline Education (years) & $9.2 \pm 3.3$ & $9.6 \pm 3.3$ & 0.492 \\
\hline ASA score of $2, n(\%)$ & $38(63.3)$ & $35(58.3)$ & 0.315 \\
\hline MMSE (point) & $25.7 \pm 1.4$ & $25.5 \pm 1.3$ & 0.343 \\
\hline Duration of anesthesia (min) & $98.5 \pm 12.3$ & $102.2 \pm 13.3$ & 0.113 \\
\hline \multicolumn{4}{|l|}{ Frequency of comorbidities: } \\
\hline Coronary artery disease, $n(\%)$ & $14(23.3)$ & $18(30)$ & 0.682 \\
\hline Diabetes, $n(\%)$ & $15(25.0)$ & $10(16.7)$ & 0.261 \\
\hline Hypertension, $n$ (\%) & $47(78.3)$ & $50(83.3)$ & 0.484 \\
\hline Cerebral infarction, $n$ (\%) & $14(23.3)$ & $19(31.7)$ & 0.307 \\
\hline \multicolumn{4}{|l|}{ Cytokines } \\
\hline$\| \mathrm{L}-1 \beta(\mathrm{pg} / \mathrm{ml})$ & $1.33 \pm 0.17$ & $1.75 \pm 0.15$ & 0.000 \\
\hline IL-6 (pg/ml) & $91.03 \pm 9.32$ & $121.44 \pm 9.58$ & 0.000 \\
\hline IL-10 (pg/ml) & $6.89 \pm 1.78$ & $5.45 \pm 1.68$ & 0.000 \\
\hline TNF-a (pg/ml) & $20.64 \pm 3.78$ & $44.03 \pm 3.52$ & 0.000 \\
\hline $\mathrm{S} 100 \beta(\mathrm{ng} / \mathrm{mL})$ & $0.26 \pm 0.09$ & $0.34 \pm 0.11$ & 0.000 \\
\hline CD14 + CD16+ monocytes (\%) & $19.70 \pm 4.57$ & $35.75 \pm 4.31$ & 0.000 \\
\hline Prevalence of delirium, $n$ (\%) & $7(11.7)$ & $23(38.3)$ & 0.001 \\
\hline
\end{tabular}

$B M I$ body mass index, ASA American Society of Anesthesiologists, MMSE mini-mental state examination. Significant results are italicized.

as HS, and CD14+CD16+ monocyte level was set as the dependent variable on the first day after the surgery. Linear regression analysis demonstrated the significant association between monocyte level and HS $(\beta=-0.61 ; 95 \% \quad C I, \quad-0.74$ to $-0.48 ; \quad P=0.000)$ (Table 4). The duration of anesthesia was associated with the level of CD14+CD16+ monocytes $(P=0.030)$.

\section{Association between prophylactic $\mathrm{HS}$ and delirium after adjustment for CD14 + CD16+ monocytes}

HS and CD14+CD16+ monocyte levels were set as the independent variables, and delirium was set as the dependent variable. The effect of HS on the probability of delirium decreased (odds ratio [OR], 0.86; 95\% CI, 0.14 to $5.33 ; P=0.874$ ) when accounting for $\mathrm{CD} 14+$

Table 3 Random-effect logistic regression model showing associations between hypertonic saline and delirium

\begin{tabular}{llll}
\hline & OR & $95 \% \mathrm{Cl}$ & $P$ value \\
\hline Hypertonic saline & 0.13 & 0.04 to 0.41 & 1.05 to 1.28 \\
Age, per year & 1.16 & 0.10 to 0.89 & .005 \\
Sex, women versus men & 0.30 & 0.49 to 4.09 & .030 \\
ASA, II versus III & 1.41 & 0.60 to 1.31 & .525 \\
MMSE, per point & 0.89 & 0.37 to 4.79 & .540 \\
Hypertension, yes versus no & 1.33 & 0.54 to 6.09 & .664 \\
Diabetes, yes versus no & 1.81 & 0.26 to 3.19 & .341 \\
Coronary artery disease, yes versus no & 0.90 & 1.28 to 11.07 & .873 \\
Cerebral infarction, yes versus no & 3.76 & 0.63 to 5.83 \\
Duration of anesthesia, $<97$ min versus $\geq 97$ min & 1.92 & .016 \\
\hline ASA American Sor
\end{tabular}

ASA American Society of Anesthesiologists, MMSE mini-mental state examination, $\mathrm{Cl}$ confidence interval, OR odds ratio The receiver operating characteristic (ROC) curve was calculated to evaluate the cut-off point of the duration of anesthesia. The patients were divided into two groups based on the cut-off point. Significant results are italicized 


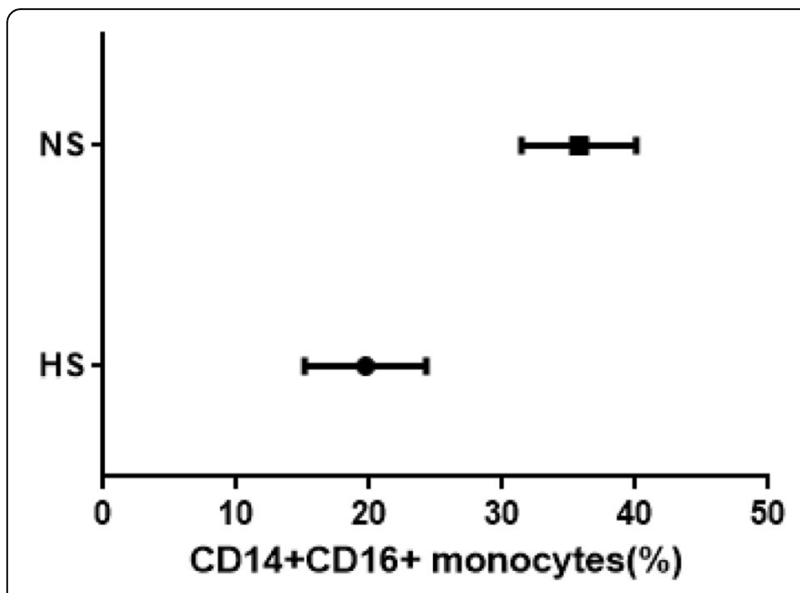

Fig. 1 Relationship between the levels of CD14 + CD16+ monocytes according to prophylactic hypertonic saline (HS) before anesthesia. Each point shows the average CD14 + CD16+ monocytes (\%) in each group along with standard deviation

CD16+ monocytes (Table 5). Delirium remained significantly related to $\mathrm{CD} 14+\mathrm{CD} 16+$ monocytes (odds ratio [OR], 14.51; 95\% CI, 2.41 to 87.38; $P=0.004$ ) (Table 5). Analysis using the multivariable logistic regression model indicated that age, sex, and cerebral infarction remained associated with the development of postoperative delirium after adjusting for other important preoperative factors (all $P<0.05$ ).

Cytokines and perioperative factors were identified using a stepwise logistic regression model and the occurrence of delirium as the dependent variable. TNF$\alpha$ remained significant after adjusting for other risk factors in the multivariate models (odds ratio [OR], 1.10; 95\% CI, 1.05 to $1.16 ; P=0.000$ ) (Table 6). The positive

Table 4 Linear regression model showing associations between hypertonic saline and CD14 + CD16+ monocytes

\begin{tabular}{llll}
\hline & $\beta$ & $95 \% \mathrm{Cl}$ & $P$ value \\
\hline Hypertonic saline & -0.61 & -0.74 to -0.48 & .000 \\
Age, per year & 0.00 & -0.00 to 0.02 & .160 \\
Sex, women versus men & -0.07 & -0.20 to 0.07 & .327 \\
ASA, II versus III & 0.05 & -0.09 to 0.18 & .486 \\
MMSE, per point & -0.03 & -0.08 to 0.01 & .166 \\
Hypertension, yes versus no & -0.06 & -0.22 to 0.10 & .464 \\
$\begin{array}{l}\text { Diabetes, yes versus no } \\
\text { Coronary artery disease, }\end{array}$ & 0.04 & -0.11 to 0.20 & .588 \\
yes versus no & -0.09 & -0.25 to 0.06 & .242 \\
Cerebral infarction, & & & .456 \\
yes versus no & 0.05 & -0.09 to 0.19 & .030 \\
$\begin{array}{l}\text { Duration of anesthesia, } \\
<97 \text { min versus } \geq 97 \text { min }\end{array}$ & 0.15 & 0.01 to 0.28 &
\end{tabular}

ASA American Society of Anesthesiologists, MMSE mini-mental state examination, $\mathrm{Cl}$ confidence interval, $\mathrm{OR}$ odds ratio. The ROC curve was calculated to evaluate the cut-off point of the duration of anesthesia. The patients were divided into two groups based on the cut-off point. Significant results are italicized
Table 5 Random-effect logistic regression model showing the relationship between hypertonic saline and delirium, adjusted by CD14 + CD16+ monocytes

\begin{tabular}{llll}
\hline & OR & $95 \%$ Cl & $P$ value \\
\hline Hypertonic saline & 0.86 & 0.14 to 5.33 & 0.874 \\
$\begin{array}{l}\text { CD14 + CD16+ monocytes, } \\
\leq 34.40 \% \text { versus }>34.40 \%\end{array}$ & 14.51 & 2.41 to 87.38 & 0.004 \\
Age, per year & 1.16 & 1.04 to 1.29 & 0.009 \\
$\begin{array}{l}\text { Sex, women versus men } \\
\text { ASA, II versus III }\end{array}$ & 0.31 & 0.09 to 0.99 & 0.048 \\
MMSE, per point & 1.40 & 0.45 to 4.37 & 0.562 \\
Hypertension, yes versus no & 1.49 & 0.38 to 5.94 & 0.569 \\
$\begin{array}{l}\text { Diabetes, yes versus no } \\
\text { Coronary artery disease, }\end{array}$ & 1.64 & 0.44 to 6.08 & 0.461 \\
$\begin{array}{l}\text { yes versus no } \\
\text { Cerebral infarction, } \\
\text { yes versus no }\end{array}$ & 1.34 & 0.35 to 5.09 & 0.669 \\
$\begin{array}{l}\text { Duration of anesthesia, } \\
<97 \text { min versus }>97 \text { min }\end{array}$ & 1.27 & 0.38 to 4.27 & 0.694 \\
\hline ASA American Society of Anesthesion 1.48 & & 0.027 \\
\hline
\end{tabular}

ASA American Society of Anesthesiologists, MMSE mini-mental state examination, $\mathrm{Cl}$ confidence interval, $O R$ odds ratio. The ROC curve was calculated to evaluate the cut-off point of CD14 + CD16+ monocytes and the duration of anesthesia. The patients were divided into two groups based on the cut-off point. Significant results are italicized

predictive ability of the multivariate logistic regression model tested by ROC analysis showed an area under the curve of 0.851 (95\% CI, 0.77 to 0.93 ) (Fig. 2).

\section{Discussion}

In this study, HS can reduce the development of postoperative delirium in geriatric patients after adjusting for age, sex, and comorbidities. The underlying mechanism may be related to the inhibition of the secretion of inflammatory factors by monocytes. The anti-inflammatory action may form a part of the basis of HS-delirium relationship, and this finding confirms the neuroinflammatory hypothesis of delirium.

Treatment with HS could be responsible for a stretching relief that could influence natriuretic and immunoinflammatory markers [19]. Liu et al. [20] also confirmed the function of HS in improving inflammation. In the present study, we investigated the relationship between HS and postoperative delirium. Systemic inflammation is a prominent feature of numerous medical and surgical

Table 6 Stepwise logistic regression model showing the relationship between cytokines and delirium

\begin{tabular}{llll}
\hline & OR & $95 \% \mathrm{Cl}$ & $P$ value \\
\hline TNF-a (pg/ml) & 1.1 & 1.05 to 1.16 & 0.000 \\
Age, per year & 1.15 & 1.04 to 1.27 & 0.006 \\
Sex, women versus men & 0.27 & 0.09 to 0.77 & 0.014 \\
Cerebral infarction, yes versus no & 2.98 & 1.05 to 8.42 & 0.039 \\
\hline
\end{tabular}




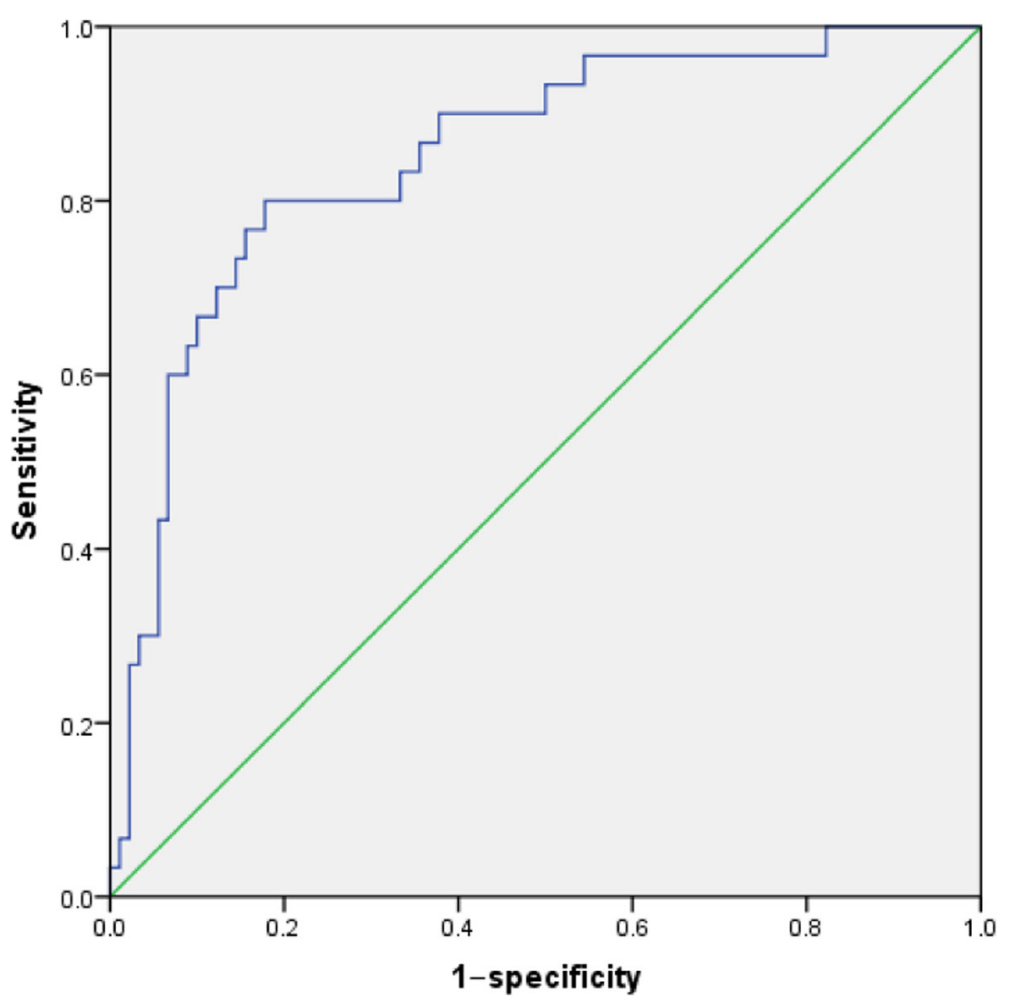

Fig. 2 Analysis of the receiver operating characteristic for the predictive value of delirium

conditions associated with delirium, particularly when these conditions involve tissue destruction or infection [6]. A strong association was found between the elevated levels of inflammation biomarkers and the risk for developing delirium $[8,11,21]$. We primarily determined the correlation of HS and postoperative delirium and identified whether or not their relationship was statistically significant. The results provide proof and principle for large trials to further study clinical outcomes.

The inflammatory and metabolic effect of saline overload correction in treatment of cirrhosis complications suggested a possible role of inflammatory and metabolicnutritional variables as severity markers in these patients [22]. Moreover, several investigations have characterized HS in terms of its specific immune-modulatory qualities, such as monocyte subset redistribution and cytokines production $[20,23]$. The activity of proinflammatory cytokines is thought to be a critical link between cognitive impairments and POD pathogenesis [24]. In addition, HS inhibits pro-inflammatory cytokine production but stimulates anti-inflammatory cytokine production by monocytes/macrophages [12]. Mahnaz et al. [25] conducted a clinical trial involving 40 patients who underwent elective coronary artery bypass grafting (CABG) surgery; those who received HS exhibited lower level of IL- 6 and higher level of IL-10 than those given with NS $48 \mathrm{~h}$ after the surgery. In addition, compared with treatment with furosemide alone, treatment with furosemide/HSS resulted in a significant lowering of plasma levels of atrial natriuretic peptide (ANP), BNP, TNF- $\alpha$, IL- 1 , and IL-6 [26]. Thus, we investigated the plasma levels of cytokines and monocytes.

Of all proinflammatory cytokines, TNF- $\alpha$ mediating immune responses is regarded as the initiating medium of systemic inflammatory responses. TNF- $\alpha$ can enhance the adhesive capacity of neutrophils and monocytes; induce the release of IL-1 $\beta$, IL- 6 , IL- 8 , and adhesion molecules; and eventually exert a cascade amplification effect on inflammation [27]. Surgery leads to the production of TNF- $\alpha$, which subsequently disrupts the blood brain barrier and causes the infiltration of inflammatory macrophages in the brain parenchyma, specifically in the hippocampus [27]. IL-1 $\beta$ plays a pivotal role in surgeryinduced cognitive dysfunction. Memory impairment caused by surgery intervention is related to the increased levels of plasma cytokines and the hippocampal neuron IL-1 $\beta$ pathway [28]. IL-6 is a critical cytokine connecting innate and acquired immunity, and is vital for induction of both peripheral and central defense against injury and inflammation [29]. In addition, IL-6 facilitates the effect of IL-1 $\beta$ in mediating inflammation and causing hippocampal-dependent memory impairment [30]. Thus, the level of pro-inflammatory cytokines, including IL-1 $\beta$, IL-6, TNF- $\alpha$, and anti-inflammatory IL-10, may be 
related to the pathophysiology of POD and can be determined by ELISA. Furthermore, cerebral or extra-cerebral neuron damage can increase the serum level of S100 $\beta$, which is a response of the brain to proinflammatory cytokines [31]. In the present study, preconditioning HS decreased the levels of IL-1 $\beta$, IL-6, TNF- $\alpha$, and S100 $\beta$ and increased the level of IL-10 compared with those in the NS group. Hence, HS significantly influenced the secretion of inflammatory and anti-inflammatory cytokines. Similarly, another study reported that HS effectively inhibits pro-inflammatory cytokines and enhances the production of counter-inflammatory cytokines [32]. It is also consistent with the previous study, in which higher $S 100 \beta$ levels were found in patients with delirium than in patients without delirium [31].

An animal study confirmed the negative effect of elevated levels of pro-inflammatory cytokines in hippocampus [33]. We also tested the association between cytokines and delirium. Multivariate logistic regression analysis demonstrated the significant association of delirium with TNF- $\alpha$ and the receiver operating characteristics analysis for the predictive value of delirium showed an area under the curve of 0.851 . Our findings support that acute systemic inflammatory TNF- $\alpha$ can sufficiently induce acute dysfunction in a cognitive task reliant on attentional and working memory function; two key cognitive domains were impaired in delirium [34]. Elevated TNF- $\alpha$ level could alter human cognitive and affective behavior. However, previous studies have had conflicting results that TNF- $\alpha$ did not associate with delirium [35]. Further studies are needed to confirm these imparities.

TNF- $\alpha$ and IL-10 are the main inflammatory cytokines and are mainly produced by monocytes and macrophages [36, 37]. Monocytes positively express HLA-DR and CD11b, and differentially express CD14 and CD16 in humans [38]. Classical monocytes (CD14++CD16-), which comprise peripheral blood monocytes, can migrate to injury and infection sites, where they differentiate into inflammatory macrophages. The subset of intermediate monocytes (CD14+CD16+) generally exhibits inflammatory characteristics [12]. The increase in the levels of monocytes $(\mathrm{CD} 14+\mathrm{CD} 16+)$ indicates the presence of systemic inflammatory responses. Rizoli et al. [12] reported that HS can inhibit inflammation by suppressing CD14 + CD16+ monocytes. Using flow cytometry analysis, we found the significantly lower level of CD14+CD16+ monocytes in patients of the HS group than those of the NS group. In addition, logistic regression analysis demonstrated the strong association between CD14+CD16+ monocytes and delirium. These results indicate that surgery caused the selective expansion of pro-inflammatory CD14 $+\mathrm{CD} 16+$ monocytes in peripheral blood, and the probability of delirium occurrence is due to $\mathrm{CD} 14+\mathrm{CD} 16+$ monocytes redistribution. Hence, HS can reduce the occurrence of POD, which might inhibit CD14+CD16+ monocytes, resulting in reduced release of inflammatory factors.

The observed effect after HS administration suggests a biologically plausible causal pathway, where the reduction in systemic inflammation mediates the relationship of HS and delirium. In the analysis, the criteria for mediation were met. Prophylactic HS (independent variable) was associated with delirium (dependent variable) and with $\mathrm{CD} 14+\mathrm{CD} 16+$ monocytes (mediating variable). $\mathrm{CD} 14+\mathrm{CD} 16+$ monocytes were associated with delirium. Adjusting the association between prophylactic HS and delirium by $\mathrm{CD} 14+\mathrm{CD} 16+$ monocytes showed a reduction in effect size. Thus, prophylactic HS could be associated with a low risk of delirium in geriatric patients who underwent hip surgery.

In this study, we identified TNF- $\alpha$, age, sex, and cerebral infarction as independent risk factors for the occurrence of POD. This finding is consistent with previous reports that age and male sex are the major risk factors for the development of delirium [39]. Age and sex, which cannot be modified through medical intervention, are demographic risk factors associated with delirium. Changes in the neurons or neurotransmitters in the brain can lead to an increased risk of cognitive disruption in patients with cerebral infarction. The relationship between acute stroke and delirium has also been well-established. Delirium may be a non-specific finding of acute stroke and may occur in the brain area affected by stroke [40]. Tuttolomondo et al. [41] suggested a possible role for a T-cell component in acute ischemic stroke clinical setting showing a different peripheral frequency of CD4 + CD28- cells in relation of subtypes of stroke and the role of CD4 + CD28- subset could represent a natural extension of cytokine, selectins, and adhesion molecule activation [42]. In addition, WBC could represent a marker of inflammatory process that is well recognized to have a role in the pathogenesis of the ischemic neuronal damage [43]. CD4 + CD28- cells and WBC may be involved in POD induced by stroke.

This study found that the incidence of delirium in elderly patients who underwent hip replacement was about $25 \%$. This finding is consistent with the results of previous studies [4]. In aged rats, peripheral stimulation induces high levels of cytokines (IL-1 $\beta$, IL-6, and TNF- $\alpha$ ) in the hippocampus and retains them for a long time [7]. The duration of delirium is an independent risk factor of postoperative cognitive dysfunction (POCD) in aged patients after surgery [10]. POCD is characterized by persistent cognitive difficulties, including memory and attention problems and executive dysfunction, and commonly occurs after the surgery. We previously demonstrated that $7.5 \% \mathrm{HS}$ can improve cognitive function after the surgery of aged rats, and the underlying mechanism may be related to the inhibition 
of hippocampal neuron apoptosis [44]. Pre-clinical experiments confirmed the relationship between the inflammation of hippocampus neurons and POCD [45]. TNF- $\alpha$ exerts robust acute effects on brain function in the degenerating brain and is highly relevant for the illness-induced exacerbations of brain dysfunction, including depression, delirium, and POCD [46]. The effect of HS on reducing hippocampal neuron apoptosis by reducing neuroinflammation to improve POCD remains to be discussed.

Patients who have pre-existing cognitive impairment are more likely to show cognitive impairment [47]. Thus, we tested MMSE with patients before the surgery to rule out confounding factors. The current research exhibits certain limitations. The number of patients is relatively small because of the strict exclusion criteria. We only enrolled 120 patients, and the CI was insufficiently precise. Despite the multiple adjustments conducted, residual confounding remains a possibility. Time between hip fracture and subsequent surgery, preoperative medications, pain, and use of opioids and other drugs postoperatively may as well affect cognitive status. The observation time is short. A long observation period may be important in determining the relationship between the subsets of delirium and the level of inflammatory factors. Prolonging the observation time might be helpful in guiding the management of delirious patients. The limitation of cognitive assessment in geriatric patients is recognized. The Nu-DESC test is designed to be administered by a bedside nurse based on clinical observations in their routine practice. This test has very high sensitivity and low specificity [16]. At present, confusion assessment method is the most widely used tool for highly sensitive and specific screening of delirium. As well as, we merely collected plasma to detect the inflammation which cannot abundantly represent its level of cerebrospinal fluid. Increased systemic, serum cytokine levels may precede an increased neuroinflammatory response associated with delirium [48]. The peripheral inflammation may have altered central nervous system cytokine profiles through several channels reportedly [49-51].

\section{Conclusion}

Prophylactic HS exerts beneficial effect on delirium in geriatric population, and such effect may be related to inhibition of monocytes. However, the favorable effects of HS on improving long-term treatment outcomes remain unknown. Further work should be conducted to elucidate the mechanisms by which HS can intervene during the development of delirium.

\section{Abbreviations}

ASA: American society of anesthesiologists; BMI: Body mass index; CABG: Coronary artery bypass grafting; $\mathrm{Cl}$ : Confidence interval; HS: Hypertonic saline; IL-1 $\beta$ : Interleukin-1 $\beta$; MMSE: Mini-mental state examination; NS: Normal saline; Nu-DESC: Nursing delirium screening scale; OR: Odds ratio; POCD: Postoperative cognitive dysfunction;
POD: Postoperative delirium; ROC: Receiver operating characteristic; TNFa: Tumor necrosis factor alpha

\section{Acknowledgements \\ We would like to thank Meng Zhao, Chunping Yin, YangYang Guo, and Fang Gao for their advice and support on the design and analysis of this project.}

\section{Funding}

This work was supported by the National Natural Science Foundation of China (no. 30972832) and Hebei Provincial Government Funded Clinical Talents Cultivation and Basic Research Projects (no. 361005). The sponsors had no role in the design of the study and collection, analysis, and interpretation of data and preparation, review, or approval of the manuscript.

\section{Availability of data and materials}

No additional data are available.

\section{Authors' contributions}

$X X$ and FX was involved in the study concept and design, data acquisition, data analysis and interpretation, study coordination and drafting and revising the manuscript. QJW contributed to the data acquisition, data interpretation, and revision of the manuscript. XGC participated in the data acquisition, study coordination, data interpretation, and revision of the manuscript. QZ and YNL contributed to the study concept and design, study coordination, data acquisition, data interpretation and drafting and revising the manuscript. SPH and CFC helped in the study design and manuscript preparation. All authors read and approved the final version of the manuscript.

\section{Ethics approval and consent to participate}

The study was approved by ethics committee of the Third Hospital of Hebei Medical University (2016-012-1), and written informed consent was obtained from all subjects.

\section{Consent for publication}

Not applicable

\section{Competing interests}

The authors declare that they have no competing interests.

\section{Publisher's Note}

Springer Nature remains neutral with regard to jurisdictional claims in published maps and institutional affiliations.

\section{Author details}

'Department of Anesthesiology, The Third Hospital of Hebei Medical University, No 139, Ziqiang Road, Shijiazhuang 050051, Hebei, People's Republic of China. ${ }^{2}$ Department of Respiration, Tianjin Institute of Respiratory Diseases, Tianjin Haihe Hospital, Tianjin Medical University, Tianjin 300350, People's Republic of China.

Received: 8 August 2017 Accepted: 8 November 2017

Published online: 14 November 2017

References

1. Page V, Navarange S, Gama S, MaAuley D. Routine delirium monitoring in a UK critical care unit. Crit Care. 2009;13(1):R16.

2. Ehlenbach WJ, Hough $\mathrm{CL}$, Crane PK, et al. Association between acute care and critical illness hospitalization and cognitive function in older adults. JAMA. 2010;303:763-70.

3. Milbrandt EB, Deppen $\mathrm{S}$, Harrison $\mathrm{PL}$, et al. Costs associated with delirium in echanically ventilated patients. Crit Care Med. 2004;32:955-62.

4. Lee $\mathrm{KH}, \mathrm{Ha}$ YC, Lee YK, Kang H, Koo KH. Frequency, risk factors, and prognosis of prolonged delirium in elderly patients after hip fracture surgery. Clin Orthop Relat Res. 2011;469:2612-20.

5. Inouye SK, Charpentier PA. Precipitating factors for delirium in hospitalized elderly persons. Predictive model and interrelationship with baseline vulnerability. JAMA. 1996;275:852-7.

6. Cerejeira J, Firmino H, Vaz-Serra A, Mukaetova-Ladinska EB. The neuroinflammatory hypothesis of delirium. Acta Neuropathol. 2010; 119(6):737-54. 
7. Giuseppe PC, Corinna B. Neuroinflammatory challenges compromise neuronal function in the aging brain: postoperative cognitive delirium and Alzheimer's disease. Behav Brain Res. 2017;322:269-79.

8. Timothy DG, Lorraine BW, Gordon RB, et al. Associations of markers of inflammation and coagulation with delirium during critical illness. Intensive Care Med. 2012;38(12):1965-73.

9. Paul R, William M, Philip O, Michael H. Review of postoperative delirium in geriatric patients undergoing hip surgery. Geriatr Orthop Surg Rehabil. 2016;7(2):100-5.

10. Androsova G, Krause R, Winterer G, Schneider R. Biomarkers of postoperative delirium and cognitive dysfunction. Front Aging Neurosci. 2015;7:112.

11. Holmes C, Cunningham C, Zotova E, Culliford D, Perry VH. Proinflammatory cytokines, sickness behavior, and Alzheimer disease. Neurology. 2011;77:212-8.

12. Rizoli SB, Rhind SG, Shek PN, et al. The Immunomodulatory effects of hypertonic saline resuscitation in patients sustaining traumatic hemorrhagic shock. Ann Surg. 2006;243(1):47-57.

13. Motaharinia J, Etezadi F, Moghaddas A, Mojtahedzadeh M. Immunomodulatory effect of hypertonic saline in hemorrhagic shock. Daru. 2015;23:47.

14. Kim JY, Choi SH, Yoon YH, Moon SW, Cho YD. Effects of hypertonic saline on macrophage migration inhibitory factor in traumatic conditions. Exp Ther Med. 2013;5(1):362-6.

15. Zeng WX, Han YL, Zhu GF, et al. Hypertonic saline attenuates expression of notch signaling and proinflammatory mediators in activated microglia in experimentally induced cerebral ischemia and hypoxic BV-2 microglia. BMC Neurosci. 2017;18:32

16. Stukenberg S, Franck M, Spies CD, Neuner B, Myers I, Radtke FM. How can postoperative delirium be predicted in advance? A secondary analysis comparing three methods of early assessment in elderly patients. Minerva Anestesiol. 2016;82:751-9.

17. Baron RM, Kenny DA. The moderator-mediator variable distinction in social psychological research: conceptual, strategic, and statistical considerations. J Pers Soc Psychol. 1986;51:1173-82.

18. MacKinnon DP, Lockwood CM, Hoffman JM, West SG, Sheets V. A comparison of methods to test mediation and other intervening variable effects. Psychol Methods. 2002;7:83-104.

19. Tuttolomondo A, Pinto A, Di Raimondo D, et al. Changes in natriuretic peptide and cytokine plasma levels in patients with heart failure, after treatment with high dose of furosemide plus hypertonic saline solution (HSS) and after a saline loading. Nutr Metab Cardiovasc Dis. 2011;21(5):372-9.

20. Liu Z, Li Y, Liu B, et al. Synergistic effects of hypertonic saline and valproic acid in a lethal rat two-hit model. Trauma Acute Care Surg. 2013;74(4):991-7.

21. Capri M, Yani SL, Chattat R, et al. Pre-operative, high-IL-6 blood level is a risk factor of post-operative delirium onset in old patients. Front Endocrinol (Lausanne). 2014;5:173

22. Tuttolomondo A, Di Raimondo D, Bellia C, et al. Immune-inflammatory and metabolic effects of high dose furosemide plus hypertonic saline solution (HSS) treatment in cirrhotic subjects with refractory ascites. PLoS One. 2016; 11(12):e0165443.

23. Junger WG, Rhind SG, Rizoli SB, et al. Resuscitation of traumatic hemorrhagic shock patients with hypertonic saline-without dextran-inhibits neutrophil and endothelial cell activation. Shock. 2012:38(4):341-50.

24. Terrando N, Eriksson LI, Ryu JK, et al. Resolving postoperative neuroinflammation and cognitive decline. Ann Neurol. 2011;70:986-95.

25. Mahnaz M, Fardin Y, Mohammad A, et al. Comparison of hypertonic saline versus normal saline on cytokine profile during CABG. Daru. 2012;20(1):49.

26. Tuttolomondo A, Pinto A, Parrinello G, Licata G. Intravenous high-dose furosemide and hypertonic saline solutions for refractory heart failure and ascites. Semin Nephrol. 2011;31(6):513-22.

27. Kalliolias GD, Ivashkiv LB. TNF biology, pathogenic mechanisms and emerging therapeutic strategies. Nat Rev Rheumatol. 2016;12(1):49-62.

28. Eleanor C, Roanna JH, Barbara CM, et al. Cerebrospinal fluid markers of neuroinflammation in delirium: a role for interleukin-1 $\beta$ in delirium after hip fracture. J Psychosom Res. 2014;77(3):219-25.

29. Jones SA. Directing transition from innate to acquired immunity: defining a role for IL-6. J Immunol. 2005:175:3463-8.
30. Baier PC, May U, Scheller J, Rose JS, Schiffelholz T. Impaired hippocampusdependent and -independent learning in IL-6 deficient mice. Behav Brain Res. 2009;200:192-6.

31. Van Munster BC, Korevaar JC, Korse CM, Bonfrer JM, Zwinderman AH, de Rooij SE. Serum S100ß in elderly patients with and without delirium. Int J Geriatr Psychiatry. 2010;25(3):234-9.

32. Gundersen $Y$, Ruud TE, Krohn CD, Sveen O, Lyngstadaas SP, Aasen AO. Impact of hypertonic saline on the release of selected cytokines after stimulation with LPS or peptidoglycan in ex vivo whole blood from healthy humans. Shock. 2010;34(5):450Y454

33. Culley BJ, Snayd M, Baxter MG, et al. Systemic inflammation impairs attention and cognitive flexibility but not associative learning in aged rats: possible implications for delirium. Front Aging Neurosci. 2014;6:107.

34. Davis DH, Skelly DT, Murray C, et al. Worsening cognitive impairment and neurodegenerative pathology progressively increase risk for delirium. Am J Geriatr Psychiatry. 2015;23(4):403-15.

35. Ritter C, Tomasi CD, Dal-Pizzol F, et al. Inflammation biomarkers and delirium in critically ill patients. Crit Care. 2014;18:R106.

36. Gushchin V, Stegalkina S, Alam HB, Kirkpatrick JR, Rhee PM, Koustova E. Cytokine expression profiling in human leukocytes exposure to hypertonic and isotonic fluids. J Trauma. 2002:52:867-71.

37. Powers KA, Woo J, Khadaroo RG, Papia G, Kapus A, Rotstein OD. Hypertonic resuscitation of hemorrhagic shock upregulates the anti-inflammatory response by alveolar macrophages. Surgery. 2003;134:312-8.

38. Thomas G, Tacke R, Hedrick CC, Hanna RN. Nonclassical patrolling monocyte function in the vasculature. Arterioscler Thromb Vasc Biol. 2015;35(6):1306-16.

39. Fortini A, Morettini A, Tavernese G, Facchini S, Tofani L, Pazzi M. Delirium in elderly patients hospitalized in internal medicine wards. Intern Emerg Med. 2014:9(4):435-41.

40. Latif D, Dursun A. Evaluation of Patients with Delirium in the Emergency Department. Balkan Med J. 2012;29(4):381-5.

41. Tuttolomondo A, Pecoraro R, Casuccio A, et al. Peripheral frequency of CD4 +CD28- cells in acute ischemic stroke: relationship with stroke subtype and severity markers. Medicine (Baltimore). 2015;94(20):e81.

42. Tuttolomondo A, Di Raimondo D, Pecoraro R, et al. Immuneinflammatory markers and arterial stiffness indexes in subjects with acute ischemic stroke. Atherosclerosis. 2010;213:311-8.

43. Tuttolomondo A, Pedone C, Pinto A, et al. Predictors of outcome in acute ischemic cerebrovascular syndromes: the GIFA study. Int J Cardiol. 2008:125(3):391-6.

44. Zhang Q, Yuan TB, Li YN, Xin X, He JH, Wang QJ. Pre-treatment with nimodipine and $7.5 \%$ hypertonic saline protects aged rats against postoperative cognitive dysfunction via inhibiting hippocampal neuronal apoptosis. Behav Brain Res. 2017:321:1-7.

45. Hovens IB, van Leeuwen BL, Nyakas C, Heineman E, van der Zee EA, Schoemaker RG. Prior infection exacerbates postoperative cognitive dysfunction in aged rats. Am J Physiol Regul Integr Comp Physiol. 2015:309(2):R14-59.

46. Hennessy E, Gormley S, Lopez-Rodriguez AB, Murray C, Murray C, Cunningham C. Systemic TNF-a produces acute cognitive dysfunction and exaggerated sicknless behavior when superimposed upon progressive neurodegeneration. Brain Behav Immun. 2016; https://doi. org/10.1016/j.bbi.2016.09.011

47. Oh ES, Li M, Fafowora TM, et al. Preoperative risk factors for postoperative delirium following hip fracture repair: a systematic review. Int J Geriatr Psychiatry. 2015;30(9):900-10.

48. Westhoff D, Witlox J, Koenderman L, et al. Preoperative cerebrospinal fluid cytokine levels and the risk of postoperative delirium in elderly hip fracture patients. J Neuroinflammation. 2013:10:122.

49. Kohman RA, Rhodes JS. Neurogenesis, inflammation and behavior. Brain Behav Immun. 2013;27(1):22-32.

50. Nguyen MD, Julien JP, Rivest S. Innate immunity: the missing link in neuroprotection and neurodegeneration? Nat Rev Neurosci. 2002:3(3):216-27

51. Perry $\mathrm{VH}$. The influence of systemic inflammation on inflammation in the brain: implications for chronic neurodegenerative disease. Brain Behav Immun. 2004;18(5):407-13 